\title{
Random Walk in Random Environment: A Counterexample?
}

\author{
Maury Bramson ${ }^{1 \star}$ and Richard Durrett ${ }^{2 \star \star}$ \\ ${ }^{1}$ School of Mathematics, University of Minnesota, Minneapolis, MN 55455, USA \\ 2 Department of Mathematics, Cornell University, Ithaca, NY 14853, USA
}

\begin{abstract}
We describe a family of random walks in random environments which have exponentially decaying correlations, nearest neighbor transition probabilities which are bounded away from 0 , and yet are subdiffusive in any dimension $d<\infty$.
\end{abstract}

\section{Introduction}

Random walks in random environments have been the subject of much attention in recent years in connection with $1 / f$ noise [1] and as disordered systems of interest in their own right. They have been studied by various nonrigorous methods: Monte Carlo studies [2], series expansions [3,4], and the renormalization group [5-7]; some special models have been analyzed rigorously $[8,9]$. Here we have cited only papers about the model in dimensions $d>1$; the literature concerning the one dimensional problem is too large to catalogue.

At this point a consensus has developed [2,3,5-7] that for a model with short range correlations, two is the upper critical dimension for the problem: for $d>2$ the mean square displacement will be asymptotically linear in time (i.e., normal diffusive behavior), while for $d<2$ the behavior is subdiffusive. The point of this paper is to describe an example which casts some doubt on the universality of the last conclusion. Specifically, we describe a family of models with spatially homogeneous random environments which have exponentially decaying correlations and nearest neighbor transition probabilities bounded away from 0 so that a random walk in any of these random environments is subdiffusive in any dimension $d<\infty$.

The models we will consider are a special case of what we have called [9] random walk on a random hillside. In these systems one starts with a random

\footnotetext{
$\star$ This author partially supported by NSF grant DMS 83-1080

$\star \star$ This author partially supported by NSF grant DMS-85-05020 and the Army Research Office through the Mathematical Sciences Institute at Cornell University
} 
function $V: \mathbb{R}^{d} \rightarrow \mathbb{R}$ (the hillside or potential), defines

$$
\alpha(x, y)=\exp [-\beta V((x+y) / 2)]
$$

for $x, y \in Z^{d}$ with $|x-y|=1$, and for convenience sets $\alpha(x, y)=0$ otherwise. The $\alpha(x, y)$ are nonnegative, so if we let

and

$$
\alpha(x)=\sum_{y} \alpha(x, y)
$$

$$
p(x, y)=\alpha(x, y) / \alpha(x)
$$

then

$$
p(x, y) \geqq 0 \quad \text { and } \quad \sum_{y} p(x, y)=1,
$$

i.e., $p$ is a transition probability. From $p$ we construct our random walk in random environment $X(n)$ in the usual way: if $X(n)=x$ (in words, the particle is at $x$ at time $n$ ), then the probability it will jump to $y$ at time $n+1$ is $p(x, y)$ independent of what happened before time $n$. To prepare for later developments the reader should note that the definition of $p$ is unchanged if we replace $\alpha$ by

$$
\bar{\alpha}(x, y)=\exp [-\beta\{V((x+y) / 2)-V(x)\}],
$$

since the extra factor will cancel when we normalize. The value of $p(x, y)$ therefore only depends on the value of the increments $V((x+\cdot))-V(x)$. In our model $V$ has slope $\leqq 1$ so for neighboring points, $p(x, y) \geqq e^{-\beta / 2} / 2 d e^{\beta / 2}$.

In an earlier paper [9] the second author, following a suggestion of Marinari et al. [1] studied the special case of this model in which " $V$ belongs to an ensemble which is invariant (at least for its long distance behavior) under the transformation $V \rightarrow V^{*}$, where

$$
V^{*}(\lambda x)-V^{*}(0)=\lambda^{\theta}(V(x)-V(0)) .
$$

He found, as they predicted, that in such a random environment the position of the random walk at time $t$ is asymptotically $(\log t)^{1 / \theta}$. (See [9] for a precise statement and proof.) This result is not, however, inconsistent with the "consensus" referred to above, because, as several people have pointed out to us [10], the correlations in the above model decay like a small power of $1 /|x|$. The last objection does not apply to our new example because its correlations in $p(x, y)$ decay exponentially fast.

To construct the random hillside $V$ which defines the model, we let $k(z), z \in Z^{d}$, be independent random variables with

$$
\begin{gathered}
P[k(z)=0]=1-\delta, \\
P[k(z)=k]=\delta \varepsilon(1-\varepsilon)^{k-1}, \quad k=1,2, \ldots,
\end{gathered}
$$

where $\varepsilon$ is a small number which will be chosen later and $0<\delta<1$ is arbitrary.

We think of $V$ as being the surface of a (random) moon, with $k(z)$ giving the radius of the crater centered at $z$. If we let $|x|=\left|x_{1}\right|+\cdots+\left|x_{d}\right|$, then the function

$$
\varphi_{k}(x)=\min \{|x|-k, 0\}
$$


describes what we mean by a (square) crater of radius $k$ centered at 0 , and we define the surface of our moon by

$$
V(x)=\min _{z} \varphi_{k(z)}(x-z),
$$

where the minimum is taken over $z$ in $Z^{d}$.

From the above definition it is clear that the increments in $V$ have exponentially decaying correlations. We have already observed that $\{p(x, y):|x-y|=1\}$ is bounded away from zero. The next result shows the third property claimed in the introduction: random walks in these random environments are subdiffusive.

Theorem 1. Suppose that $\varepsilon \leqq 1 / 2,0<\delta<1$, and $N>0$. If $\beta \geqq 2(N+d+1)$, then

$$
P\left[\max _{m \leqq n}|X(m)| \geqq n^{1 / N}\right] \rightarrow 0 \quad \text { as } n \rightarrow \infty .
$$

If one takes, for example, $\varepsilon=1 / 2, d=3$ and $N=4$, the last theorem implies that when $\beta \geqq 16$ the RWRE is subdiffusive for all values of $\delta>0$. The reason for the bound $n^{1 / N}$ is in spirit as follows: The largest hole that the particle falls into before leaving the ball of radius $r$ will be of order $c \log r$, where $c=-2 \log r / \log (1-\varepsilon)$ for $d \geqq 2$. The time it takes to climb out of this hole will be for order $e^{c \beta \log r}=r^{c \beta}$; the time it takes to leave the ball will be at least as large. Inverting, one obtains the above bound, but under the somewhat stronger assumption $\beta \geqq 2(N+d+1)$.

The reader (especially one familiar with an earlier version of this paper) may at this point expect a diatribe about the failure of nonrigorous methods. We do think that this example should make the reader think twice before asserting that renormalization drives all models to the weak disorder fixed point. However, the simple fact is that it does not contradict any claims in the physics literature. As a referee has pointed out, the models in the references have random forces rather than the random potentials we consider here. (See ref. 7 for more on this point.) We think that if the random variables $p(x, \cdot)$ are i.i.d., then it is probably correct that the corresponding RWRE is diffusive in $d>2$. Hence the last character of the title.

Returning to the mathematical aspects of the result above, we note that a result of DeMasi, Ferrari, Goldstein, and Wick [11,12] implies

Theorem 2. If

$$
E[\exp (-\beta V(0))]<\infty,
$$

then the random walk in the random environment is diffusive.

Combining this result with Theorem 1 shows that for any $\varepsilon \leqq 1 / 2$ and $\delta>0$ there is phase transition from diffusive to subdiffusive behavior as $\beta$ increases. A conjecture which implies the existence of a critical value and gives its location is that in the models considered here, $E[\exp (-\beta V(0))]=\infty$ implies that the RWRE is subdiffusive.

\section{Demonstration of Theorem 1}

We continue to use the notation employed in Sect. 1. We define $\varphi_{k}, V, \alpha, p$ and $X$ as before. Denote by $\mathscr{V}$ the $\sigma$-algebra generated by $V$. Note that conditioned 
on $V, X(n)$ is a reversible Markov chain with (infinite) equilibrium measure $\alpha(x)$. Except when specified otherwise, $X(0)=0$ is assumed. As usual, $\Omega$ will denote the probability space and $\omega$ its elements.

Set $B(r)=\left\{x \in \mathbb{Z}^{d}:|x|<r\right\}$. As $r$ increases, $B(r)$ will with high probability contain deeper and more numerous craters. A particle executing the motion $X(n)$ should on occasion fall into such deep craters. Owing to (1), it should take a long time before returning to the surface (where $V=0$ ), or for that matter, to any fixed level $g(\varepsilon, d)$. Craters deeper than $g(\varepsilon, d)$, for appropriate $g(\varepsilon, d)$, compose a comparatively small portion of $\mathbb{Z}^{d}$, and so the particle should typically not be able to move far until it returns to this level. The last three statements can in fact be justified and will form the basis for our proof of Theorem 1 .

To be more explicit, introduce $a_{i}$ and $b_{i}$ with

$$
a_{i}=[\log i], \quad b_{i}=a_{3}+\cdots+a_{i},
$$

for $i \geqq 3$, with $[w]$ denoting the integer part of $w \in \mathbb{Z}$. From $b_{i}$, define the sets

$$
B_{i}=\left\{x \in \mathbb{Z}^{d}:|x|<b_{i}\right\}
$$

and $A_{i}=B_{i}-B_{i-1}$. Since we are unable to say much about the motion of $X(n)$, crude arguments regarding the placement of deep craters are required. In Proposition 1, we give a lower bound on the probability that before leaving $B_{i}$, $X(n)$ falls at least to depth $a_{i}$ in a prescribed manner. Although this probability is small, it is not too much smaller than $p_{a_{l}}$, and the event will occur with probability close to one for some $B_{i}$ satisfying $B(r / 4) \leqq B_{i} \leqq B(r / 2)$, if $r$ is large. In Proposition 2 , a lower bound is given for the time it takes for a particle to climb up to level $g$ from a prescribed depth $h$. The time will typically be exponential in $\beta(h+g)$. For $h=a_{i}$ and given $N$, reference to (5) shows that this will be of greater magnitude than $r^{N}$ for $\beta$ chosen large enough. The last step is provided by Proposition 3 . There it is shown that, except for a very small probability, no sites in $B(r / 2)$ are connected to $B^{c}(r)$ except through paths which reach level $g$; if $\delta$ is small enough, one can set $g=0$. Combining Propositions $1-3$, we therefore see that with a probability close to one, a particle will fall at least to depth $a_{i}$, and then take at least $r^{N}$ units of time before exiting $B(r)$, if $r$ is large. Inverting, we obtain Theorem 1.

We will find it useful to define

$$
A(x)=\left\{z: \varphi_{k(z)}(x-z)=V(x)\right\}
$$

if $V(x)<0$. We will then say that " $x$ is influenced by $A(x)$ ". Note that for $|y-x|=1$, $V(y)=V(x)-1$ iff $|y-z|=|x-z|-1$ for some $z \in A(x)$. In this case, $A(y) \subset A(x)$.

Lemma 1. Fix $V, h, x_{0}$, and $y$, and suppose that $x_{0}$ is influenced by $A$ with $\operatorname{dist}\left(A, x_{0}\right) \geqq h$. For $\beta \geqq 2 \log 6 d$ and $X(0)=x_{0}$,

$$
P[V(X(j))=V(X(0))-j, \quad j=1, \ldots, h] \geqq(3 / 4)^{h} .
$$

Proof. Let $\mathscr{P}_{m}$ denote the set of paths $\left(x_{0}, \ldots, x_{m}\right)$ (i.e., $\left.\left|x_{j}-x_{j-1}\right|=1\right)$ with $V\left(x_{j}\right)=V\left(x_{0}\right)-j$ for $j=1, \ldots, m$. For given $\left(x_{0}, \ldots, x_{m-1}\right) \in \mathscr{P}_{m-1}, m \leqq h$, let

$$
B=\left\{x_{m}:\left(x_{0}, \ldots, x_{m}\right) \in \mathscr{P}_{m}\right\} \text {. }
$$


Since $x_{0}$ is influenced by $A$ and $\operatorname{dist}\left(A, x_{0}\right) \geqq h, B$ is not empty. Note that

$$
V\left(x_{m}\right) \geqq V\left(x_{m-1}\right) \text { if } x_{m} \notin B \text {. }
$$

Therefore if $\beta \geqq 2 \log 6 d$,

$$
\sum_{x_{m} \in B} p\left(x_{m-1}, x_{m}\right) \geqq|B| e^{\beta / 2} /\left(2 d+|B| e^{\beta / 2}\right) \geqq 3 / 4 .
$$

Inequality (7) follows by induction.

We will find it convenient to introduce two variants of $V(x)$. Let

$$
\begin{aligned}
V_{i}(x) & =\min _{z \in B_{i}} \varphi_{k(z)}(x-z), \\
\tilde{V}_{i}(x) & =V_{i}(x) \wedge\left(\operatorname{dist}\left(\partial B_{i}, x\right)-a_{\imath}\right) .
\end{aligned}
$$

Equations (8) will be used in Proposition 1 in the context of $\sigma_{i}$ (defined below). Also, for Proposition 1 and later results, let

$$
\begin{aligned}
T_{\Lambda} & =\min \{n: X(n) \in \Lambda\}, \quad \Lambda \subset \mathbb{Z}^{d}, \\
T_{x}^{1} & =\min \{n \neq 0: X(n)=x\}, \quad x \in \mathbb{Z}^{d}, \\
T_{x}^{m} & =\min \left\{n>T_{x}^{m-1}: X(n)=x\right\}, \quad m \geqq 2, \\
T_{i} & =\min \left\{n:|X(n)|=b_{i}\right\},
\end{aligned}
$$

and

$$
\begin{aligned}
\sigma_{i} & =T_{i} \wedge \min \left\{n:|X(n)|>b_{i-1}, V_{i}(X(n)) \neq \tilde{V}_{i}(X(n))\right\}, \\
\tau_{i} & =\min \left\{n: V(X(n)) \leqq-a_{i}\right\} .
\end{aligned}
$$

(If a set is empty, assign the value $\infty$.) Note that under fixed $V$, these are all stopping times. Lastly, define

$$
G_{i}=\left\{\omega: \tau_{i} \leqq T_{i}\right\}
$$

and

$$
\mathscr{G}_{i}=\sigma\left(G_{1}, \ldots, G_{i}\right), \quad \mathscr{V}_{i}=\sigma\left(V,\left\{X(n): n \leqq \sigma_{i}\right\}\right) .
$$

The idea behind the proof of Proposition 1 is that at least with the probability given in (13), there is some $z$ with $|z|=b_{i}$ and $k(z)=a_{i}$, and which satisfies (14). If $V\left(X\left(\sigma_{i}\right)\right)>-a_{i}$, then $X\left(\sigma_{i}\right)$ is influenced by $z$ or some other point not in $B_{i}$, and one can apply Lemma 1 .

Proposition 1. For $G_{i}, \mathscr{G}_{i-1}$ as defined in (11)-(12) and $\beta \geqq 2 \log 6 d$,

$$
P\left[G_{i} \mid \mathscr{G}_{i-1}\right] \geqq \delta \varepsilon(3(1-\varepsilon) / 4)^{a_{2}} .
$$

Proof. Choose $Z_{i}$ so that $\left|Z_{i}\right|=b_{i}$ and

$$
\left|Z_{i}-X\left(\sigma_{i}\right)\right|=b_{i}-\left|X\left(\sigma_{i}\right)\right| .
$$

Since $V(X(n))$ can decrease by at most 1 in each unit of time, on

$$
K_{i}=\left\{\omega: k\left(Z_{i}\right)=a_{i}\right\},
$$


one has

$$
T_{i} \geqq \sigma_{i}+a_{i}-V\left(X\left(\sigma_{i}\right)\right)
$$

So for

$$
F_{i}=K_{i} \cap\left\{V\left(X\left(\sigma_{i}+j\right)\right)=V\left(X\left(\sigma_{i}\right)\right)-j, \quad j=1, \ldots, a_{i}-V\left(X\left(\sigma_{i}\right)\right)\right\},
$$

one has

$$
F_{i} \subset G_{i}
$$

Set

$$
F_{i}\left(x_{0}\right)=K_{i}\left(x_{0}\right) \cap\left\{\omega: V\left(X^{\prime}(j)\right)=V\left(x_{0}\right)-j, \quad j=1, \ldots, a_{i}-V\left(x_{0}\right)\right\},
$$

where $X^{\prime}(j)$ is a copy of $X(j)$ with $X^{\prime}(0)=x_{0}$ and $K_{i}\left(x_{0}\right)$ is a copy of $K_{i}$ corresponding to $X^{\prime}$ with $x_{0}$ substituted for $X\left(\sigma_{i}\right)$ in (14). (At $x_{0}=X\left(\sigma_{i}\right), K_{i}\left(x_{0}\right)=K_{i}$.) Conditioned on $\mathscr{V}, X(n)$ is strong Markov, and so

$$
P\left[F_{i} \mid \mathscr{V}_{i}\right]=P\left[F_{i}\left(X\left(\sigma_{i}\right)\right) \mid \mathscr{V}\right]
$$

$\left(=\sum_{x_{0}} P\left[F_{i}\left(x_{0}\right) \mid \mathscr{V}\right] P\left[X\left(\sigma_{i}\right)=x_{0}\right]\right)$. Since $\mathscr{G}_{i-1} \subset \mathscr{V}_{i},(16)$ and (17) imply that

$$
P\left[G_{i} \mid \mathscr{G}_{i-1}\right] \geqq E\left[P\left[F_{i}\left(X\left(\sigma_{i}\right)\right) \mid \mathscr{V}\right] \mid \mathscr{G}_{i-1}\right] .
$$

Owing to the definition of $\sigma_{i}, X\left(\sigma_{i}\right)$ is, on $K_{i}$, influenced by some $A$ with $A \cap B_{i}=\phi$ or $V\left(X\left(\sigma_{i}\right)\right)<-a_{i}$. Since

$$
\operatorname{dist}\left(A, X\left(\sigma_{i}\right)\right) \geqq\left|Z_{i}-X\left(\sigma_{i}\right)\right|,
$$

one can in the first case apply Lemma 1 with $h=\left|Z_{i}-X\left(\sigma_{i}\right)\right|$, where $0<h<a_{i}$. It follows that on $K_{i}$,

$$
P\left[F_{i}\left(X\left(\sigma_{i}\right)\right) \mid \mathscr{V}\right] \geqq \sum_{h=0}^{a_{i}-1}(3 / 4)^{h} P\left[\left|Z_{i}-X\left(\sigma_{i}\right)\right|=h \mid \mathscr{V}\right]>(3 / 4)^{a_{2}}
$$

(Off $K_{i}$, the left-hand side of (19) of course equals zero.) One can check that $\mathscr{G}_{i-1}$ and $K_{i}$ are independent. One therefore obtains from (18) and (19) that

$$
P\left[G_{i} \mid \mathscr{G}_{i-1}\right] \geqq(3 / 4)^{a_{i}} P\left[K_{i}\right]=\delta \varepsilon[3(1-\varepsilon) / 4]^{a_{2}} .
$$

The proof of Proposition 2 makes use of the following lemma, which was suggested to us by J. Baxter. (One may also use the Dirichlet Principle.) Here, $Y(n)$ is a positive recurrent Markov chain with transition probabilities $q(x, y)$, $x, y \in \mathbb{Z}^{d}$, and equilibrium probability measure $\pi(x)$. We introduce

$$
f_{x y}=P_{x}\left[T_{x}^{1}>T_{y}\right]=P[Y \text { visits } y \text { before returning to } x \mid Y(0)=x] .
$$

Lemma 2. Assume $Y(n)$ is positive recurrent with a single recurrence class. Then for all $x, y$,

$$
\pi(x) f_{x y}=\pi(y) f_{y x} .
$$

Proof. Equation (20) is well known (cf. Chung [13], page 50, Theorem 5). Perhaps the most intuitive proof runs as follows. Fix $x, y$, and set

$$
u(z)=P[Y \text { visits } y \text { before } x \mid Y(0)=z]
$$


for arbitrary state $z$. Then $u(x)=0, u(y)=1$, and $u$ is harmonic off $\{x, y\}$, i.e.,

$$
u(z)=\sum_{w} q(z, w) u(w) \text { for } z \neq x, y .
$$

Also,

$$
u(x)=\sum_{w} q(x, w) u(w)-f_{x y}, \quad u(y)=\sum_{w} q(y, w) u(w)+f_{y x} .
$$

Summing over (21) and (22) with weights $\pi(z)$, one obtains

$$
\begin{aligned}
\sum_{z} \pi(z) u(z) & =\sum_{z} \pi(z)\left[\sum_{w} q(z, w) u(w)\right]-\pi(x) f_{x y}+\pi(y) f_{y x} \\
& =\sum_{w} u(w)\left[\sum_{z} \pi(z) q(z, w)\right]-\pi(x) f_{x y}+\pi(y) f_{y x} \\
& =\sum_{w} \pi(w) u(w)-\pi(x) f_{x y}+\pi(y) f_{y x} .
\end{aligned}
$$

The assertion follows immediately.

Below, we let $H^{g}(x)$ denote "the hole below $g$ " containing $x$, that is, the set of sites $y$ connected to $x$ by some path $\left(x_{0}, \ldots, x_{m}\right)$ with $x_{0}=x, x_{m}=y$ and $V\left(x_{j}\right)<g$ for $0 \leqq j \leqq m$. Also, let

$$
\partial H^{g}(x)=\left\{y \notin H^{g}(x): \exists z \in H^{g}(x) \text { with }|y-z|=1\right\} .
$$

(For such $z, V(z)=g-1$.) $g=g(\varepsilon, d) \leqq 0$ and will be chosen later. When convenient, we write $H, \partial H$.

The proof of Proposition 2 consists of fixing $V$, and applying Lemma 2 to the Markov chain induced by restricting $\mathbb{Z}^{d}$ to $H \cup \partial H$.

Proposition 2. Fix $V$. For $w \in \mathbb{Z}^{d}$ with $V(w) \leqq-h<g$,

$$
P_{w}\left[T_{\partial H^{q}(w)} \leqq n\right] \leqq n\left|\partial H^{g}(w)\right| e^{\beta(1-h-g)} .
$$

Proof. Construct the Markov chain $\tilde{X}(n)$ with state space $\bar{H}^{g}(w)=H^{g}(w) \cup\{\Delta\}$ and transition probabilities $\tilde{p}(x, y)$ as follows. Set

$$
\begin{aligned}
& \tilde{\alpha}(x, y)=\alpha(x, y) \text { for } x, y \in H, \\
& \tilde{\alpha}(x, \Delta)=\tilde{\alpha}(\Delta, x)=\sum_{y \in \hat{c} H} \alpha(x, y) \text { for } x \in H, \\
& \tilde{\alpha}(\Delta, \Delta)=\sum_{x, y \in \hat{H} H} \alpha(x, y), \\
& \tilde{\alpha}(x)=\sum_{y \in \bar{H}} \tilde{\alpha}(x, y) \text { for } x \in \bar{H},
\end{aligned}
$$

and

$$
\tilde{p}(x, y)=\tilde{\alpha}(x, y) / \tilde{\alpha}(x) \text { for } \quad x, y \in \bar{H} .
$$

The Markov chain thus defined is positive recurrent and reversible with equilibrium measure $\tilde{\alpha}(x)$. It is easy to see that on $\left\{n: n \leqq T_{\partial H}\right\}, \widetilde{X}$ may be obtained from $X$ by collapsing the sites in $\partial H(w)$ to $\Delta$. Consequently,

$$
P_{w}\left[T_{w}^{1}>T_{\partial H}\right]=P_{w}\left[\tilde{T}_{w}^{1}>\tilde{T}_{\Delta}\right],
$$


where $T$ and $\tilde{T}$ are the stopping times corresponding to $X$ and $\tilde{X}$.

We now apply Lemma 2 with $Y=\tilde{X}$. The stationary distribution $\pi$ is a constant multiple of $\tilde{\alpha}$ and so

$$
\tilde{\alpha}(x) / \tilde{\alpha}(y)=\pi(x) / \pi(y) \text { for } \quad x, y \in \bar{H} .
$$

It follows from (20) that

$$
P_{w}\left[\tilde{T}_{w}^{1}>\tilde{T}_{\Delta}\right]=P_{\Delta}\left[\tilde{T}_{\Delta}^{1}>\tilde{T}_{w}\right] \tilde{\alpha}(\Delta) / \tilde{\alpha}(w) \leqq \tilde{\alpha}(\Delta) / \tilde{\alpha}(w) .
$$

One can check from the definition of $\alpha(x)$ that

$$
\tilde{\alpha}(w) \geqq 2 d e^{-\beta / 2} \cdot e^{\beta h}, \quad \tilde{\alpha}(\Delta) \leqq 2 d e^{\beta / 2} \cdot e^{-\beta g} \cdot|\partial H|,
$$

and so the left-hand side of (25) is at most

$$
|\partial H| e^{\beta(1-h-g)} .
$$

Together with (24), this shows that

$$
P_{w}\left[T_{w}^{1}>T_{\partial H}\right] \leqq|\partial H| e^{\beta(1-h-g)} .
$$

Iterating, one obtains

$$
P_{w}\left[T_{w}^{n}>T_{\partial H}\right] \leqq n|\partial H| e^{\beta(1-h-g)} .
$$

Since $T_{w}^{n} \geqq 2 n$,

$$
P_{w}\left[T_{\partial H}<2 n\right] \leqq n|\partial H| e^{\beta(1-h-g)} .
$$

The next two lemmas will be used in proving Proposition 3.

Lemma 3. For $\gamma>0$,

$$
P\left[\max (k(z)-\rho)>r^{\gamma}: z \in B(r+\rho), \rho \in \mathbb{Z}^{+}\right] \leqq C_{1} \exp \left\{-r^{\gamma / 2}\right\},
$$

where $C_{1}$ depends on $\gamma, \varepsilon$, and $d$.

Proof. The left-hand side of (27) is at most

$$
\sum_{\rho=0}^{\infty}(2(r+\rho))^{d} \delta \varepsilon(1-\varepsilon)^{R+\rho} \leqq(4 r)^{d} \varepsilon(1-\varepsilon)^{R} \sum_{\rho=0}^{\infty}(1-\varepsilon)^{\rho}+4^{d} \varepsilon(1-\varepsilon)^{R} \sum_{\rho=0}^{\infty} \rho^{d}(1-\varepsilon)^{\rho},
$$

with $R=\left[r^{\nu}\right]$. The first term on the right-hand side equals

$$
(4 r)^{d}(1-\varepsilon)^{R} \text {. }
$$

The second term is at most

$$
4^{d} \varepsilon(1-\varepsilon)^{R} \sum_{\rho=1}^{\infty} \rho(\rho+1) \cdots(\rho+d-1)(1-\varepsilon)^{\rho-1}=d !(4 / \varepsilon)^{d}(1-\varepsilon)^{R} .
$$

Their sum is at most

$$
C_{1} \exp \left\{-r^{\gamma / 2}\right\}
$$

for appropriate $C_{1}$ (which depends on $\gamma, \varepsilon$, and $d$ ).

In order to obtain bounds on the size of the hole $H^{g}(x)$, we let $H_{0}(x)=\{x\}$, 
and inductively define $H_{j}(x)$ as the set of $y \in \mathbb{Z}^{d}$ such that for some $w \in H_{j-1}(x)$ and $z \in \mathbb{Z}^{d}$

$$
\varphi_{k(z)}(y-z)<g \text { and } \varphi_{k(z)}(w-z)<g .
$$

Since $y$ is connected to $w$ through a path which never reaches the level $g, H_{j}(x)$ consists of those sites in $H^{g}(x)$ which are at most " $j$ craters removed" from $x$. Also, set

and

$$
h_{j}(x)=H_{j}(x)-H_{j-1}(x)
$$

$$
J(x)=\max \left\{j: h_{j}(x) \neq \phi\right\} .
$$

Lemma 4. Suppose that

$$
g(\varepsilon, d) \leqq \frac{1}{\varepsilon} \log \left((\varepsilon / 2)^{2 d+1} / \delta(2 d) !\right)
$$

Then for $x \in \mathbb{Z}^{d}$,

$$
P[J(x) \geqq j] \leqq 1 / 2^{j} .
$$

Proof. Two points $y$ and $x$ which are connected to each other by a crater centered at $z$ must each be within distance $k(z)+g-1$ of $z$. For specified $x$, the possible number of craters of depth $k$ centered at such $z$ and the number of such $y$ located in each such crater are each at most $(2(k+g)-1)^{d}$. One therefore has the bound

$$
\begin{aligned}
E\left[\left|h_{1}(x)\right|\right] & \leqq \sum_{l=1}^{\infty}(2 l-1)^{2 d} \delta \varepsilon(1-\varepsilon)^{l-g-1} \\
& \leqq 2^{2 d} \delta \varepsilon(1-\varepsilon)^{-g} \sum_{l=1}^{\infty} l(l+1) \cdots(l+2 d-1)(1-\varepsilon)^{l-1}
\end{aligned}
$$

which equals $(2 d) ! \delta(2 / \varepsilon)^{2 d}(1-\varepsilon)^{-g}$. So,

$$
E\left[\left|h_{1}(x)\right|\right] \leqq 1 / 2
$$

for $g(\varepsilon, d)$ satisfying (29). Now,

$$
E\left[\left|h_{j}(x)\right| \mid h_{j-1}(x)\right] \leqq \sum_{z \in h_{j-1}(x)} E\left[\left|h_{1}(z)\right|\right] .
$$

By (31), this is at most

$$
\frac{1}{2}\left|h_{j-1}(x)\right|
$$

Consequently,

$$
P[J(x) \geqq j] \leqq E\left[\left|h_{j}(x)\right|\right] \leqq \frac{1}{2} E\left[\left|h_{j-1}(x)\right|\right] \leqq 1 / 2^{j}
$$

for all $j$.

For Proposition 3, we introduce

$$
E_{r}=\left\{\omega: B^{c}(r) \cap H^{g}(x)=\phi \text { for all } x \in B(r / 2)\right\} .
$$

That is, $E_{r}$ is the event that no hole (below $g$ ) which is partially contained inside $B(r / 2)$ extends outside $B(r)$. From now on, we assume that $g$ is chosen so that (29) 
holds. If $\delta$ is chosen small enough relative to $\varepsilon$ and $d$, one can in fact set $g=0$. The inequality (33) below follows from Lemmas 3 and 4, which place bounds on the size of individual craters and on the number of consecutive craters in a particular hole.

Proposition 3. For $E_{r}$ as defined above,

$$
P\left[E_{r}^{c}\right] \leqq C_{2} \exp \left\{-r^{1 / 4}\right\},
$$

where $C_{2}$ depends on $\varepsilon$ and $d$.

Proof. Denote by $L_{r}(x)$ the event that the hole (below $g$ ) containing $x$ has radius at least $r / 2$, that is,

$$
L_{r}(x)=\left\{\omega:|x-y| \geqq r / 2 \text { for some } y \in H^{g}(x)\right\},
$$

and set

$$
L_{r}=\bigcup_{x \in B(r / 2)} L_{r}(x)
$$

Clearly,

$$
E_{r}^{c} \subset L_{r}
$$

One has

$$
P\left[L_{r}\right] \leqq P\left[L_{r} \cap M_{r}\right]+P\left[M_{r}^{c}\right]
$$

where

$$
M_{r}=\left\{\omega: \max (k(z)-\rho) \leqq \sqrt{r}, z \in B(r+\rho), \rho \in \mathbb{Z}^{+}\right\} .
$$

By Lemma 3,

$$
P\left[M_{r}^{c}\right] \leqq C_{1} \exp \left\{-r^{1 / 4}\right\} .
$$

To bound the first term on the right-hand side of (35), note that for $x \in B(r / 2)$,

$$
L_{r}(x) \cap M_{r} \subset\{\omega: J(x) \geqq \sqrt{r} / 4\},
$$

where $J$ is defined in (28). Therefore,

$$
P\left[L_{r} \cap M_{r}\right] \leqq \sum_{x \in B(r / 2)} P\left[L_{r}(x) \cap M_{r}\right] \leqq \sum_{x \in B(r / 2)} P[J(x) \geqq \sqrt{r} / 4] .
$$

By Lemma 4, this is at most

$$
r^{d} / 2^{\sqrt{r} / 4}
$$

Formulas (34)-(38) show that

$$
P\left[E_{r}^{c}\right] \leqq r^{d} / 2^{\sqrt{r} / 4}+C_{1} \exp \left\{-r^{1 / 4}\right\} \leqq C_{2} \exp \left\{-r^{1 / 4}\right\}
$$

for appropriate $C_{2}$ (which depends on $\varepsilon$ and $d$ ).

We now prove Theorem 1 by using Propositions $1-3$ as sketched at the beginning of the section. 
Theorem 1. Suppose that $\varepsilon \leqq 1 / 2,0<\delta<1$, and $N>0$. If $\beta \geqq 2(N+d+1)$, then

$$
P\left[\max _{m \leqq n}|X(m)| \geqq n^{1 / N}\right] \rightarrow 0 \quad \text { as } \quad n \rightarrow \infty .
$$

Proof. Fix $r$ and set

$$
\begin{aligned}
l & =\min \left\{i: b_{i} \geqq r / 4\right\}, \\
L & =\max \left\{i: b_{i}<r / 2\right\} .
\end{aligned}
$$

It is easy to check that for $i \leqq L$,

$$
a_{i} \leqq \log r
$$

and consequently

$$
l \geqq \frac{r}{4 \log r}, \quad L-l \geqq \frac{r}{4 \log r}-1 .
$$

Now, repeated application of Proposition 1 shows that

$$
P\left[\bigcap_{i=l}^{L} G_{i}^{c}\right] \leqq \prod_{i=l}^{L}\left[1-\delta \varepsilon(3(1-\varepsilon) / 4)^{a_{l}}\right] \leqq \exp \left[-\sum_{i=l}^{L} \delta \varepsilon(3(1-\varepsilon) / 4)^{a_{i}}\right] .
$$

Plug in (40) and (41), and note that $\log (3(1-\varepsilon) / 4)>-1$ for $\varepsilon \leqq 1 / 2$. This shows that (42) is at most

$$
\exp \left[-\delta \varepsilon\left(\frac{r}{4 \log r}-1\right) r^{\log [3(1-\varepsilon) / 4]}\right] \leqq \exp \left\{-\delta \varepsilon r^{\eta}\right\}
$$

for large enough $r$ and appropriate $\eta>0$. Let

$$
G=\left(\bigcup_{i=l}^{L} G_{i}\right) \cap E_{r}
$$

Application of Proposition 3 allows one to replace (42)-(43) by

$$
P\left[G^{c}\right]=P\left[\left(\bigcap_{i=l}^{L} G_{i}^{c}\right) \cup E_{r}^{c}\right] \leqq \exp \left\{-\delta \varepsilon r^{\eta}\right\}
$$

for large enough $r$ (depending on $\varepsilon$ and $d$ ) and a new choice of $\eta$.

We restrict our attention to $X(n)$ on $G$. On this set,

$$
\tau_{i} \leqq T_{i} \text { for some } l \leqq i \leqq L
$$

Denote by $I$ the first such $i$, and set $Y_{I}=X\left(\tau_{I}\right)$. From the definition of $\tau$ and $a$,

$$
V\left(Y_{I}\right) \leqq-a_{I} \leqq-[\log l]
$$

Also,

$$
H^{g}\left(Y_{I}\right) \subset B(r)
$$

and so

$$
\left|\partial H^{g}\left(Y_{I}\right)\right| \leqq(2 r)^{d}
$$


We will denote by $\mu_{V}$ the subprobability measure on $B(r)$ induced by $Y_{I}$ (restricted to $G$ ) for fixed $V$ and by $X^{\prime}(m)$ a copy of $X(m)$ with initial distribution given by $\mu_{V}$. One can apply Proposition 2 together with (46) and (48) to obtain

$$
P_{\mu_{V}}\left[T_{\partial H\left(X^{\prime}(0)\right)} \leqq n \mid V\right] \leqq n(2 r)^{d} \exp \{\beta(1-[\log l]-g)\} .
$$

(Here the superscript $g$ is suppressed.) The right-hand side does not depend on $V$. Setting $n=r^{N}, N$ fixed, and plugging in (41), one gets

$$
P_{\mu_{V}}\left[T_{\partial H\left(X^{\prime}(0)\right)} \leqq r^{N} \mid V\right] \leqq(2 r)^{N+d-\beta / 2} \leqq 1 / r
$$

for large enough $r$ if $\beta \geqq 2(N+d+1)$. Let $T_{\partial H\left(Y_{I}\right)}^{\prime}$ denote the first time greater than $\tau_{I}$ at which $X$ hits $\partial H\left(Y_{I}\right)$. By (45) and (47),

$$
T_{\partial H\left(Y_{I}\right)}^{\prime} \leqq T_{\partial B(r)}
$$

under $X(0)=0$. Also, for fixed $V, X$ is strong Markov. Therefore,

$$
P\left[\left\{T_{\partial B(r)} \leqq r^{N}\right\} \cap G\right] \leqq P\left[\left\{T_{\partial H\left(Y_{I}\right)}^{\prime} \leqq r^{N}\right\} \cap G\right] \leqq 1 / r .
$$

Inequalities (44) and (49) together show that

$$
P\left[T_{\partial B(r)} \leqq r^{N}\right] \leqq r^{-1}+\exp \left\{-\delta \varepsilon r^{\eta}\right\} \rightarrow 0 \quad \text { as } \quad r \rightarrow \infty .
$$

Inverting, one obtains

$$
P\left[\max _{m \leqq n}|X(m)| \geqq n^{1 / N}\right] \rightarrow 0 \quad \text { as } \quad n \rightarrow \infty .
$$

\section{References}

1. Marinari, E., Parisi, G., Ruelle, D., Windey, P.: Random walk in a random environment and $1 / f$ noise. Phys. Rev. Lett. 50,1223 (1983); and On the interpretation of $1 / f$ noise. Commun. Math. Phys. 89, 1 (1983)

2. Luck, J.M.: A numerical study of diffusion and conduction in a $2 \mathrm{D}$ random medium. J. Phys. A 17, 2069 (1984)

3. Derrida, B., Luck, J.M.: Diffusion on a random lattice: Weak-disorder expansion in arbitrary dimension. Phys. Rev. B 28, 7183 (1983)

4. Winter, C. L., Newman, C. M., Neuman, S. P.: A perturbation expansion for diffusion in a random velocity field. SIAM J. Appl. Math. 44, 411 (1984)

5. Luck, J. M.: Diffusion in a random medium: A renormalization group approach. Nucl. Phys. B 225, 169 (1983)

6. Fisher, D.: Random walks in random environments. Phys. Rev. A 30, 960 (1984)

7. Fisher, D.: Random walks in two-dimensional random environments with constrained drift forces. Phys. Rev. A 31, 3841 (1985)

8. A number of systems have been analyzed by G. C. Papanicolaou and co-authors, but in all cases the behavior is diffusive in any dimension. For a survey and list of references see his article: Diffusions and random walks in random media. It has appeared in the mathematics and physics of disordered media. Hughes, B. D., Ninham, B. W., (eds). Lecture Notes in Mathematics Vol. 1035, Berlin, Heidelberg, New York: Springer (1983).

9. Durrett, R.: Multidimensional random walks in random environments with subclassical limiting behavior. Commun. Math. Phys. 104, 87 (1986)

10. Newman, C. M., Spohn, H., Spencer, T. each pointed this out to us

11. de Masi, A., Ferrari, P. A., Goldstein, S., Wick, D.: Invariance principle for reversible Markov processes with application to diffusion in the percolation regime. Contemp. Math. 41, 71 (1984) 
12. de Masi, A., Ferrarı, P.A., Goldstein, S., Wıck, D.: An invarıance princıple for reversıble Markov processes. Applications to random motions in random environments, preprent

13. Chung, K. L.: Markov chains with stationary transition probabilities. Berlin, Heidelberg, New York: Springer 1967

Communicated by M. Aizenman

Received January 7, 1988; in revised form March 18, 1988 
